Article

\title{
Development of a Tet-On Inducible Expression System for the Anhydrobiotic Cell Line, Pv11
}

\author{
Shoko Tokumoto ${ }^{1, \dagger}$, Yugo Miyata ${ }^{2,+}{ }^{+}$, Kengo Usui ${ }^{3}$, Ruslan Deviatiiarov ${ }^{4}$, Takahiro Ohkawa ${ }^{3}$, \\ Sabina Kondratieva ${ }^{4}$, Elena Shagimardanova ${ }^{4}$, Oleg Gusev ${ }^{4,5} \odot$, Richard Cornette ${ }^{1}$, \\ Masayoshi Itoh ${ }^{5}$, , Yoshihide Hayashizaki ${ }^{5}$ and Takahiro Kikawada $1,2, * \mathbb{C}$ \\ 1 Graduate School of Frontier Sciences, The University of Tokyo, Kashiwa 277-8562, Japan; \\ 4914616910@edu.k.u-tokyo.ac.jp (S.T.); cornette@affrc.go.jp (R.C.) \\ 2 Institute of Agrobiological Sciences, National Institute of Agriculture and Food Research \\ Organization (NARO), Tsukuba 305-0851, Japan; miyatay431@affrc.go.jp \\ 3 RIKEN Center for Integrative Medical Sciences (IMS), Yokohama 230-0045, Japan; \\ kengo.usui@riken.jp (K.U.); takahiro.ohkawa@riken.jp (T.O.) \\ 4 Institute of Fundamental Medicine and Biology, Kazan Federal University, Kazan, Tatarstan 420012, Russia; \\ ruselusalbus@gmail.com (R.D.); sabinakondr@gmail.com (S.K.); ryukula@gmail.com (E.S.); \\ gaijin.ru@gmail.com (O.G.) \\ 5 RIKEN Preventive Medicine and Diagnosis Innovation Program, Wako 351-0198, Japan; \\ maitoh@riken.jp (M.I.); yoshihide.hayashizaki@riken.jp (Y.H.) \\ * Correspondence: kikawada@affrc.go.jp; Tel.: +81-(29)-8386170 \\ + Contributed equally to this work.
}

Received: 8 October 2020; Accepted: 9 November 2020; Published: 11 November 2020

check for updates

Simple Summary: Anhydrobiosis is a unique ametabolic state that is characterized by extreme body water loss due to desiccation, generally over $95 \%$. The sleeping chironomid, Polypedilum vanderplanki, is an anhydrobiotic insect inhabiting semi-arid regions in Africa. Pv11 is an anhydrobiotic insect cell line established from the P. vanderplanki larvae, and the cell line also displays the extreme desiccation tolerance. This unique feature could be useful for developing a dry preservation technique. However, effective expression systems for protein production have not been available due to a limited number of experimental tools in Pv11 cells. To solve this problem, we identified a minimal promoter from the genome sequence of $P$. vanderplanki because promoters designed for model organisms are not available in Pv11 cells, although minimal promoters are commonly used for molecular biology experiments and biotechnology fields. The identification of the minimal promoter allowed us to develop an effectively inducible expression system in Pv11 cells. By using the system, several proteins including a functional protein with complex structures were successfully expressed in the cells. The successful establishment of the inducible system on Pv11 cells could contribute to bio-industrial protein production.

Abstract: The Pv11 cell line established from an African chironomid, Polypedilum vanderplanki, is the
only cell line tolerant to complete desiccation. In Pv11 cells, a constitutive expression system for
Pv11 cells was previously exploited and several reporter genes were successfully expressed. Here we
report the identification of an effective minimal promoter for Pv11 cells and its application to the
Tet-On inducible expression system. First, using a luciferase reporter assay, we showed that a 202 bp
deletion fragment derived from the constitutively active 121 -promoter functions in Pv11 cells as an
appropriate minimal promoter with the Tet-On inducible expression system. The AcGFP1 (Aequorea
coerulescens green fluorescent protein) was also successfully expressed in Pv11 cells using the inducible
system. In addition to these reporter genes, the avian myeloblastosis virus reverse transcriptase $\alpha$
subunit (AMV RT $\alpha$ ), which is one of the most widely commercially available RNA-dependent DNA
polymerases, was successfully expressed through the inducible expression system and its catalytic
activity was verified. These results demonstrate the establishment of an inducible expression system 
in cells that can be preserved in the dry state and highlight a possible application to the production of large and complex proteins.

Keywords: anhydrobiosis; desiccation tolerance; Polypedilum vanderplanki; insect cells; minimal promoter; Tet-On system

\section{Introduction}

Recombinant proteins have many applications in the biotechnology and biomedical industries. The production of recombinant proteins of sufficient quantity and quality for commercial applications relies on the selection of an appropriate combination of the gene expression system and host cell [1]. Insect cells are one of the most common hosts for recombinant protein expression because they can produce larger and more complex proteins, whose biological activities are maintained, than is possible in bacterial cells [2].

Many gene expression systems for insect cells have been developed over recent years [3-5]. For example, insect cell-based expression systems are used for the commercial production of various veterinary and human vaccines [6]. Generally, insect cells are cultured at an ambient temperature without a $\mathrm{CO}_{2}$ incubator, and thus are more cost-effective hosts than mammalian cells. To date, more than 1100 insect cell lines have been established according to the ExPASy Cellosaurus database (http://web.expasy.org/cellosaurus). However, relatively few of these are regularly used as protein expression systems, and the potential of other cell lines for protein expression has not been fully explored, especially in the case of non-model insect cell lines. Thus, using a cell line with specific features (e.g., low-temperature tolerance [7]) would benefit the development of innovative biotechnologies for protein production.

Pv11 is an insect cell line established from embryos of the African chironomid, Polypedilum vanderplanki, an insect known for its ability to undergo anhydrobiosis, a form of extreme desiccation tolerance [8,9]. Similarly, Pv11 cells also display extreme desiccation tolerance: This is induced by treatment with high concentrations of trehalose, a naturally occurring disaccharide well-known to act in many organisms as a chemical protectant against environmental abiotic stress, including desiccation [10]. Furthermore, trehalose-treated Pv11 cells express the genes associated with desiccation tolerance, such as LEA protein genes [11], which protect biomolecules from desiccation damage [12,13]. We previously showed that the induction of desiccation tolerance with trehalose in Pv11 cells allowed effective in vivo preservation of exogenously expressed enzymes, where catalytic activity was retained after over one year in the dry state [14]. These results suggest that the unique stress tolerance of Pv11 cells could be applied to new dry-preservation techniques for biomedical and bioindustrial use.

Exogenous gene expression systems are classified into two main types: Constitutive and inducible. Generally, constitutive expression systems employ constitutively active promoters, i.e., the expression of associated genes is not regulated. In Pv11 cells, the $5^{\prime}$-proximal region from -1269 to $+64 \mathrm{bp}$ relative to the transcription start site of the $P v .00443$ gene has been identified as one of the strongest constitutive promoters, namely the 121-promoter; in appropriate constructs, this has enabled Pv11 cells to constitutively express several exogenous reporter genes, such as those encoding green fluorescent protein (GFP) and luciferase [15]. Constitutive expression systems are useful for the synthesis of recombinant proteins in large quantities; however, excessive overexpression of exogenous proteins can repress cell growth and even cause cell death [16]. In contrast, inducible expression systems produce exogenous proteins only when required. In such systems, even relatively cytotoxic exogenous proteins can be produced, while minimizing their deleterious effects on cell growth. In addition, some inducible expression systems can synthesize recombinant proteins at expression levels comparable to those of major constitutive expression systems $[17,18]$. These advantages suggest that inducible 
expression systems should be prioritized for the recombinant production of any protein, regardless of its cytotoxicity. However, inducible expression systems have not yet been developed for Pv11 cells.

One of the most commonly available inducible expression platforms is the Tet-On expression system, which consists of two main factors: A reversible tetracycline-regulated transactivator (rtTA) and a tetracycline-responsive promoter element (TRE) sequence [19]. In the presence of tetracycline or derivatives such as doxycycline (Dox), rtTA is activated, binds to the TRE, and subsequently induces the expression of an associated target gene. The TRE is composed of Tet operator (TetO) sequences selectively fused to a minimal promoter suitable for the host cell [20-22].

Minimal promoters are exploited to reduce undesirable leaky expression of recombinant proteins without Dox induction in the Tet-On expression system. In general, a minimal promoter contains DNA regions that recruit the transcriptional initiation machinery, and comprises several functional subregions termed core elements or motifs, such as the TATA box, initiator (Inr), downstream core promoter element (DPE), and bridge $[23,24]$. These elements and motifs mediate the recruitment of TATA-binding protein (TBP) and TBP-associated factors [25]. Whereas several minimal promoters have been identified and designed for model organisms such as mouse and fruit fly [26], equivalent minimal promoters for most non-model organisms, including P. vanderplanki, have yet to be constructed. This represents a bottleneck for the construction of workable Tet-On expression systems for Pv11 cells.

Here we describe the identification of a minimal promoter from a deletion fragment of the 121-promoter of P. vanderplanki that is applicable to the Tet-On inducible expression system in Pv11 cells. To establish the proof-of-concept that commercially valuable proteins can be expressed using this approach, we successfully expressed avian myeloblastosis virus reverse transcriptase (AMV RT) using the Tet-On system in Pv11 cells. These results indicate the potential of Pv11 cells for bioindustrial applications.

\section{Materials and Methods}

\subsection{Cell Culture}

Pv11 cells were originally established in our laboratory [27]. The cell culture was maintained as described [15]. Briefly, Pv11 cells were cultured using IPL-41 medium (Thermo Fisher Scientific, Waltham, MA, USA) supplemented with $2.6 \mathrm{~g} / \mathrm{L}$ tryptone phosphate broth (Becton, Dickinson and Company, Franklin Lakes, NJ, USA), 10\% (v/v) fetal bovine serum (MP Biomedicals, Santa Ana, CA, USA) and $0.05 \%(v / v)$ of an antibiotic and antimycotic mixture (penicillin, amphotericin $\mathrm{B}$, and streptomycin; Merck KGaA, Darmstadt, Germany). As trehalose treatment, the cells were incubated in trehalose mixture (600 mM trehalose (Hayashibara Co., Ltd., Okayama, Japan) containing 10\% (v/v) IPL-41 medium).

\subsection{Vector Construction}

To construct the pTetO-CMV-AcGFP1 vector, the AcGFP1 gene (Takara Bio, Shiga, Japan) was cloned and amplified using the Prime STAR Max Premix (Takara Bio) with specific primers (Table S1) from pP121K-AcGFP1 [15] and inserted between the SalI and BglII sites of pTRE3G (Takara Bio) using the NEBuilder HiFi DNA Assembly Mater Mix (New England BioLabs, Ipswich, MA, USA). To construct pTetO-202bp-Nluc and pTetO-266bp-Nluc, the 202 and 266 bp promoters were cloned and amplified with specific primers (Table S1) from the pP121K-AcGFP1 series [15] and inserted into the pTetO-CMV-AcGFP1 vector digested with SalI and BglII.

To construct the luciferase Nluc expression vectors (pTetO-CMV-Nluc, pTetO-202bp-Nluc, and pTetO-266bp-Nluc), the AcGFP1 genes in the corresponding vectors were replaced by the Nluc gene. The Nluc gene was cloned and amplified using the Prime STAR Max Premix (Takara Bio) with specific primers (Table S1) from pNL1.1 (Promega, Fitchburg, WI, USA) and inserted between the Sall and BglII sites using the NEBuilder HiFi DNA Assembly Master Mix. To construct the promotor-less vector (pTetO-Nluc), the pTetO-266bp-Nluc was digested with BgIII and EcoRV to remove the 266 bp-promoter 
region. Then, the region was replaced with nonsense oligonucleotides generated by annealing the following sequences: $5^{\prime}$-agcttGGCAATCCGGTACTGTTGGTAAAGCCAg- ${ }^{\prime}$ as the sense strand; $5^{\prime}$-tcgacTGGCTTTACCAACAGTACCGGATTGCCa-3' as the anti-sense strand.

pP121K-AcGFP1 [15] was modified to make an empty vector by digestion with BamHI and SacII, and replacement of the AcGFP1 sequence with annealed oligonucleotides that introduced new restriction sites for HindIII and XhoI (sense: 5'-gatccaagcttctcgagCCGC-3', antisense: 5'-GGctcgagaagcttg-3'). We named the empty vector pPv121-MCS (Figure S1 and Data S1). For the pPv121-Tet-On 3G expression vector, $\mathrm{pPv121-MCS}$ was digested with BamHI and SacII, and the fragment for Tet-On 3G (the third generation reverse tetracycline-controlled transactivator; Takara Bio), which was prepared by PCR with specific primers (Table S1), was inserted using the NEBuilder HiFi DNA Assembly Master Mix. For the pPv121-Nluc expression vector, pTRE3G (Takara Bio) was digested with XhoI and SalI, and the Nluc fragment, which was prepared by PCR with specific primers (Table S1), was inserted using the NEBuilder HiFi DNA Assembly Master Mix. For construction of the luc2-reference vector (pPv632bp-luc2), a 632 bp fragment of the 121-promoter [15] was amplified by PCR (Table S1) and inserted between the BgIII and HindIII sites of pGL4.10 (Promega) using the NEBuilder HiFi DNA Assembly Master Mix.

A DNA fragment for the AMV RT $\alpha$ gene was synthesized by Eurofins Genomics K.K., Tokyo, Japan. For the pPv121-AMV RT $\alpha$ expression vector, AMV RT $\alpha$ gene was then amplified by PCR with specific primers (Table S1) and inserted into pPv121-MCS digested with BamHI and SacII using the NEBuilder HiFi DNA Assembly Mater Mix. For the pTetO-202bp-AMV RT $\alpha$ expression vector, the AMV RT $\alpha$ sequence was amplified and inserted into pTetO-202bp-Nluc digested with SalI and BgIII using the NEBuilder HiFi DNA Assembly Master Mix.

\subsection{Transfection and Luciferase Reporter Assay}

The cells used in each experiment were seeded at a density of $3 \times 10^{5}$ cells per $\mathrm{mL}$ into a $25 \mathrm{~cm}^{2}$ cell culture flask and grown at $25^{\circ} \mathrm{C}$ for 6 days before transfection. Transfection into Pv11 cells was carried out using a NEPA21 Super Electroporator (Nepa Gene, Ichikawa, Chiba, Japan) as described previously [28]. For transient expression of luciferase, a mixture of $2 \mu \mathrm{g}$ of either Nluc-reporter vector or 121-Nluc expression vector, $10 \mu \mathrm{g}$ luc2-reference vector, and $0.5 \mu \mathrm{g}$ Tet-On 3G expression vector was transfected into the cells (Table S2). One day after transfection, the medium was replaced with IPL-41 or trehalose mixture with or without doxycycline (Dox) at $1 \mu \mathrm{g} / \mathrm{mL}$. The cells were collected $24 \mathrm{~h}$ later, and luciferase activity was measured using an ARVO multi-label plate reader (PerkinElmer, Waltham, MA, USA) with the Nano-Glo Dual-Luciferase Reporter Assay System (Promega). Each assay was performed with 5 replicates.

\subsection{Prediction of Core Promoter Elements}

To identify putatively functional motifs in the $202 \mathrm{bp}$ minimal promoter, the Elements Navigation Tool (http://lifefaculty.biu.ac.il/gershon-tamar/index.php/element-description) [29] was used with default settings.

\subsection{GFP Expression Using the Tet-On System}

For transient expression of GFP, three combinations of the corresponding vectors were transfected into Pv11 cells as follows: $10 \mu \mathrm{g}$ pPv121-MCS; $1 \mu \mathrm{g}$ pPv121-Tet-On 3G and $9 \mu \mathrm{g}$ pTetO-202bp-AcGFP1; $1 \mu \mathrm{g}$ pPv121-MCS and $9 \mu \mathrm{g}$ pPv121-AcGFP1 (Table S2). One day after transfection, the medium was replaced with IPL-41 or trehalose mixture with or without Dox at $1 \mu \mathrm{g} / \mathrm{mL}$. After $24 \mathrm{~h}$, the AcGFP1 fluorescence in the cells was viewed using a Biozero BZ-X700 microscope (Keyence, Osaka, Japan). AcGFP1 expression was evaluated by western blot analysis. 


\subsection{AMV RT $\alpha$ Expression Using the Tet-On System}

For transient expression of AMV RT $\alpha$, three combinations of the vectors were transfected into Pv11 cells as follows: $10 \mu \mathrm{g}$ pPv121-MCS; $1 \mu \mathrm{g}$ pPv121-Tet-On 3G and $9 \mu \mathrm{g}$ pTetO-202bp-AMV RT $\alpha ; 1 \mu \mathrm{g}$ pPv121-MCS and $9 \mu \mathrm{g}$ pPv121-AMV RT $\alpha$ (Table S2). One day after transfection, the same treatment was performed as described above. Twenty-four hours after the treatment, AMV RT $\alpha$ expression in the cells was evaluated by western blot analysis, and reverse transcriptase (RT) activity was measured.

\subsection{Antibodies and Western Blot Analysis}

As primary antibodies, monoclonal anti-GFP antibody (M048-3, Medical and Biological Laboratories, Nagoya, Japan; dilution ratio: 1/1000) and monoclonal anti-His-tag antibody (D291-3, Medical and Biological Laboratories; dilution ratio: 1/2000) were used to detect AcGFP1 and AMV RT $\alpha$, respectively. Cells were lysed with radio-immunoprecipitation assay (RIPA) lysis buffer (Nacalai Tesque, Kyoto, Japan) for $30 \mathrm{~min}$ at $4{ }^{\circ} \mathrm{C}$. After centrifugation, aliquots of the supernatant were subjected to protein quantification with a Pierce BCA Protein Assay Kit (Thermo Fisher Scientific) and SDS-PAGE using a TGX Stain-Free FastCast Acrylamide Kit (Bio-Rad, Hercules, CA, USA). For AcGFP1 detection, $3 \mu \mathrm{g}$ protein/lane was used, while for AMV RT $\alpha$ detection, $15 \mu \mathrm{g}$ protein/lane was used for SDS-PAGE. After transferring to a PVDF membrane using a Trans-Blot Turbo (Bio-Rad), the membrane was blocked with $1 \%$ skimmed milk in Tris-buffered saline (TBS) with $0.1 \%$ Tween 20 (TBST) at room temperature for $1 \mathrm{~h}$. Primary antibodies were diluted in a Can Get Signal Solution1 (TOYOBO, Osaka, Japan), and incubated at room temperature for $1 \mathrm{~h}$. The membrane was washed three times with TBST for $10 \mathrm{~min}$ each wash, followed by incubation of horseradish peroxidase (HRP)-conjugated secondary antibodies (goat anti-mouse IgG, 81-6720, Thermo Fisher Scientific; dilution ratio: 1/2000) in a Can Get Signal Solution 2 (TOYOBO) at room temperature for $1 \mathrm{~h}$. The membrane was washed three times with TBST (10 min each wash), and chemiluminescent signals from ECL Prime Detection Reagents (Cytiva, Little Chalfont, Buckinghamshire, UK) were captured on a ChemiDoc ${ }^{\mathrm{TM}}$ Touch Imaging System (Bio-Rad). The membranes were stained with ponceau-S (Merck KGaA) validation (Figure S2).

\subsection{Reverse Transcriptase Assay}

Cells were lysed with a PBS (Merck KGaA)-based lysis buffer containing 0.5\% (v/v) Nonidet P-40, $10 \%(\mathrm{w} / \mathrm{v})$ glycerol, $5 \mathrm{mM}$ DTT, and Complete Mini EDTA-free Protease Inhibitor Cocktail (Merck $\mathrm{KGaA}$ ), and sonicated for $30 \mathrm{~s}$ (UR-21P, TOMY SEIKO, Tokyo, Japan). After centrifugation, aliquots of the supernatant were diluted with nuclease-free water and subjected to DNA determination using a Qubit 2.0 fluorometer and a Qubit dsDNA HS quantitation assay (Thermo Fisher Scientific). All samples were diluted to $0.436 \mathrm{ng} / \mu \mathrm{L}$ with lysis buffer and subjected to a reverse transcriptase assay (EnzChek ${ }^{\mathrm{TM}}$ Reverse Transcriptase Assay Kit; Thermo Fisher Scientific). Recombinant AMV RT $\alpha$ (Nippon Gene, Tokyo, Japan) was used to prepare a standard curve. The samples and recombinant AMV RT $\alpha$ were diluted with lysis buffer, then mixed with a reaction mixture and incubated at $25^{\circ} \mathrm{C}$. After $1 \mathrm{~h}$ incubation, the RT reactions were stopped by adding EDTA. Then, fluorescence from DNA-RNA hetroduplexes binding with PicoGreen dye was quantified using an ARVO multi-label plate reader (PerkinElmer). To eliminate the fluorescent value of PicoGreen intercalated to genomic DNA, the value of the control sample (the cells transfected the empty vector) was subtracted as a background from each sample. Each assay was performed with triplicates.

\subsection{Statistical Analysis}

All data were expressed as mean \pm SD. Statistical significance among more than two groups was examined by ANOVA followed by a Tukey post hoc test. A $p$-value $<0.05$ denoted a statistically significant difference. GraphPad Prism 8 Software (GraphPad, San Diego, CA, USA) was used for the statistical analyses. 


\section{Results}

3.1. Identification of a Minimal Promoter for the Development of an Effective Inducible Expression System in Pv11 Cells Based on the Tet-On System

To develop an effective inducible expression system, we first attempted to identify an efficient minimal promoter in Pv11 cells. We previously suggested that deletion fragments of the 121-promoter, including fragments 266 and 202 bp in length, might be candidates for a minimal promoter because such fragments have low activity in a luciferase assay [15]. Thus, we carried out a luciferase assay using vectors harboring either one of these candidates, or the cytomegalovirus (CMV) minimal promoter, or no promoter, fused with the TetO sequence to evaluate whether these fragments could work as a minimal promoter in Pv11 cells. The pPv121-Nluc vector containing the fully functional 121-promoter was used as a positive control for this assay. As reported for other insect cells [30,31], the CMV minimal promoter did not promote luciferase activity in the presence of Dox in Pv11 cells (Figure 1A and Table S3). In contrast, under standard culture conditions without Dox treatment, the $266 \mathrm{bp}$ fragment resulted in leaky expression of luciferase, while the $202 \mathrm{bp}$ fragment showed deficient transcriptional activity comparable to that of negative controls, such as pNluc, pTetO-Nluc, and pTetO-CMV-Nluc (Figure 1A and Table S3). Dox treatment enhanced luciferase expression in cells transfected with the vectors harboring the 266 and 202 bp fragments (3- and 16-fold induction, respectively) (Figure 1A and Table S3). These results indicate that, of the two fragments tested, the $202 \mathrm{bp}$ fragment functions best as a minimal promoter in the Tet-On inducible system in Pv11 cells.

Pv11 cells display extreme desiccation tolerance when induced by highly concentrated (600 mM) trehalose solution [8]. Trehalose treatment is known to trigger specific transcriptional regulatory networks that activate anhydrobiosis-related genes [32], suggesting that trehalose might affect the expression level of the exogenous Tet-On inducible system. Thus, we conducted luciferase assays for the corresponding vectors in trehalose-treated Pv11 cells. The results showed that trehalose treatment did not interfere with the inducibility of the Tet-On system in Pv11 cells (Figure 1B and Table S3). In particular, Dox treatment of Pv11 cells transfected with the vector harboring the $202 \mathrm{bp}$ fragment augmented luciferase expression 11-fold (Figure 1B). Therefore, in Pv11 cells, the Tet-On inducible system including the $202 \mathrm{bp}$ fragment (Figure 1C) as a minimal promoter can be used both during normal culture and during induction of desiccation tolerance by trehalose.

The 202 bp minimal promoter fragment contained functional core elements, such as TATA box, Inr, DPE, and bridge elements, similarly to the CMV minimal promoter (Figure 1C), although the nucleotide sequences of these elements were different in each promoter. This suggests that mammalian promoters, such as the CMV minimal promoter, may not function in Pv11 cells due to sequence differences in core promoter elements.

\subsection{AcGFP1 Expression Using the Tet-On System in Pv11 Cells}

To assess the versatility of the Tet-On system, we expressed the AcGFP1 gene in Pv11 cells. We tested this with the pTetO-202bp-AcGFP1 vector, constructed as described in the methods, using pPv121-MCS (empty vector) and pPv121-AcGFP1 as negative and positive controls, respectively. AcGFP1 fluorescence was observed in Dox-treated cells transfected with pTetO-202bp-AcGFP1 vectors under both normal culture (IPL-41 medium) and trehalose treatment conditions. In contrast, only a few AcGFP1-positive cells were observed in the absence of Dox (Figure 2A). Western blotting showed a massive accumulation of AcGFP1 protein in Dox-treated groups transfected with the pTetO-202bp-AcGFP1 vector under both IPL-41 medium and trehalose treatment conditions, while almost no AcGFP1 protein was detected in the absence of Dox (Figure 2B and Figure S2). Together with the luciferase assay data (Figure 1A,B), these results show that the Tet-On system with the $202 \mathrm{bp}$ minimal promoter can be used for the inducible expression of reporter genes, including GFP and luciferase, in Pv11 cells. 

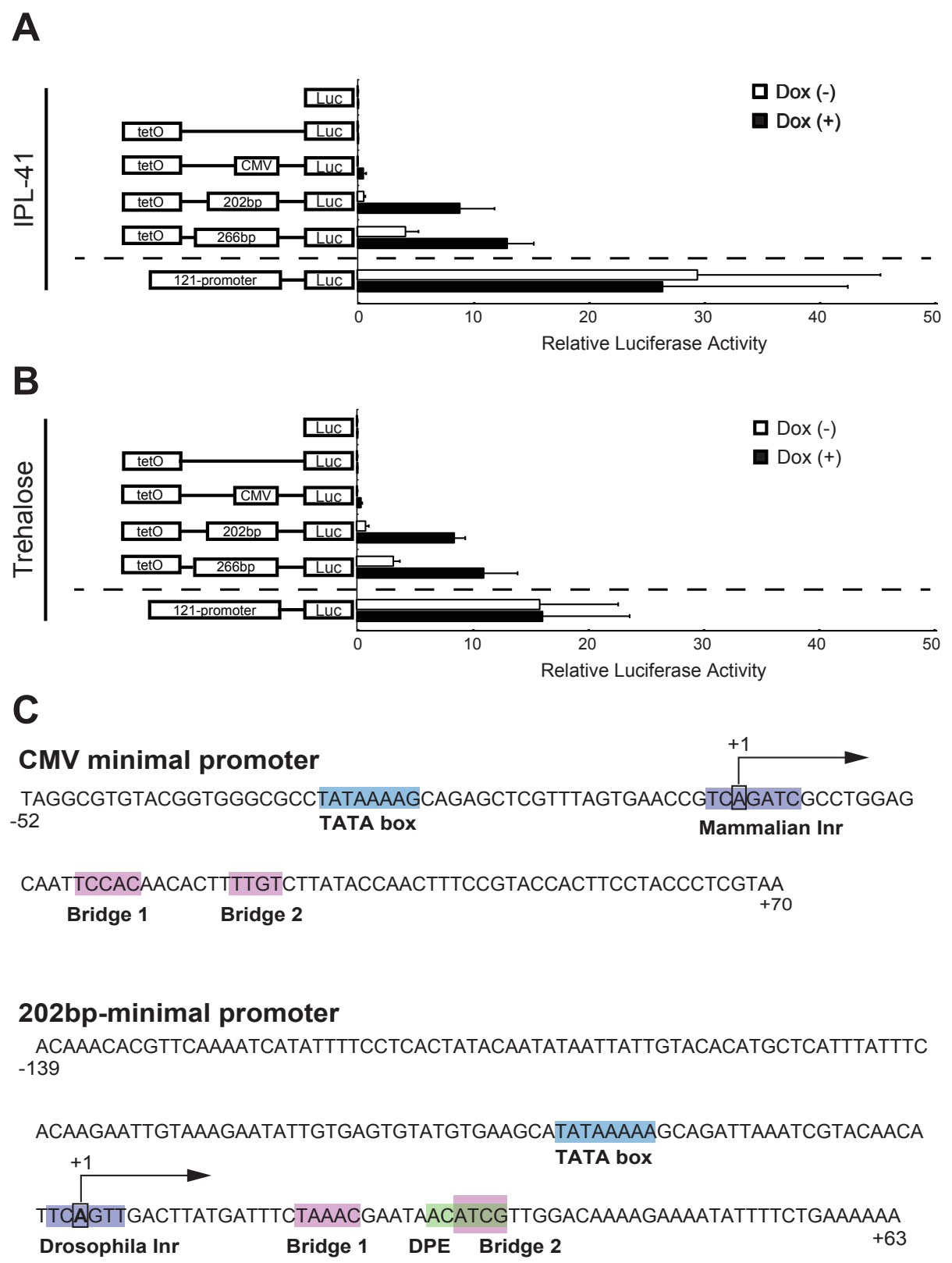

Figure 1. Identification of a minimal promoter for an inducible expression system in Pv11 cells. Luciferase activity was measured in vector-transfected Pv11 cells cultured under standard conditions (IPL-41 medium; (A)) or with $600 \mathrm{mM}$ trehalose treatment (B). A scheme of vector constructs is shown on the left and relative values in the luciferase assay are shown on the right. Normalized values are expressed as means \pm standard deviations (SD). Each assay was performed with 5 replicates. (C) The predicted core promoter elements of the cytomegalovirus (CMV) and $202 \mathrm{bp}$ minimal promoters. It is predicted that the 202 bp-promoter has Inr that closely resembles Drosophila one. Inr, initiator; DPE, downstream promoter element. TATA box, Inr, Bridge, and DPE are highlighted with blue, purple, red, and green, respectively. Transcription start sites are with +1 and black arrow. The core promoter prediction program, the Elements Navigation Tool, was used with a default setting.

\subsection{AMV RTa Expression and Measurement of RT Activity}

To examine whether Pv11 cells, like other insect cells, can express functional proteins with complex structures, such as proteins containing multiple domains, we focused on AMV RT, which is one of the most widely used RNA-dependent DNA polymerases for medical diagnoses [33] and basic research. AMV RT is a heterodimer consisting of $\alpha(63 \mathrm{kDa})$ and $\beta(95 \mathrm{kDa})$ subunits [34], but the $\alpha$-subunit alone 
has RT activity [35]. Therefore, we chose AMV RT $\alpha$ to establish the proof-of-concept that commercially valuable proteins can also be expressed in Pv11 cells.

A

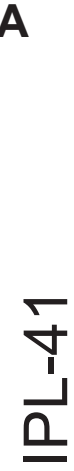

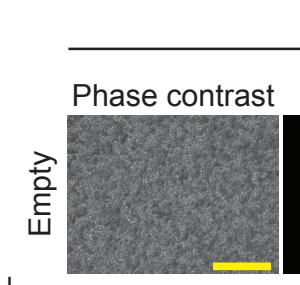

Dox (-)

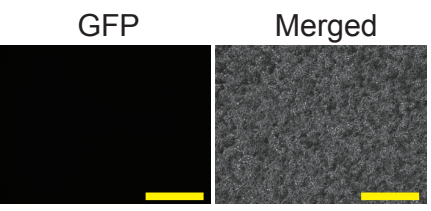

1
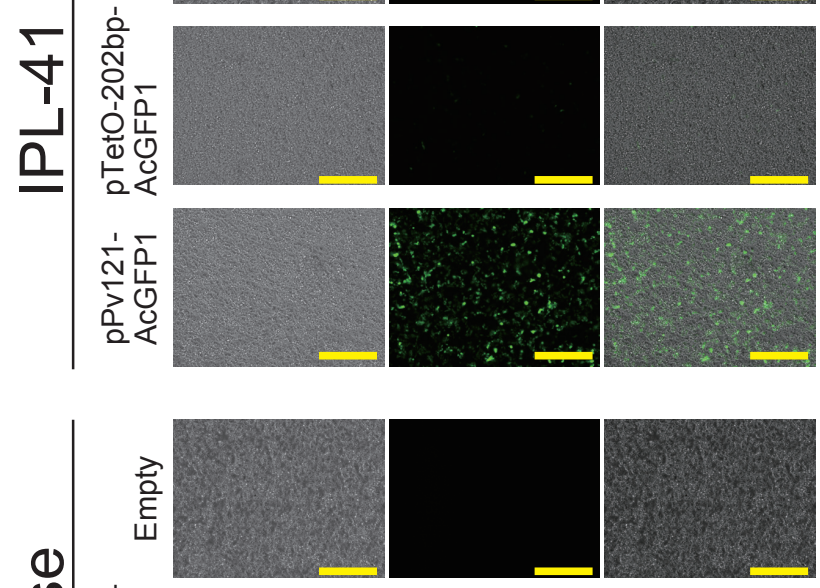

号
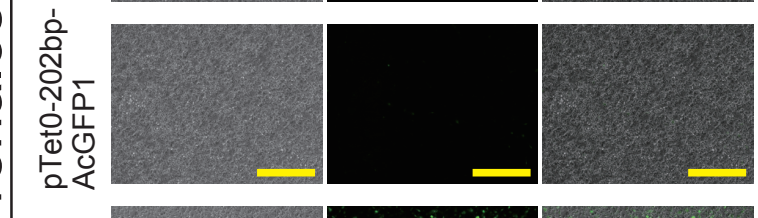
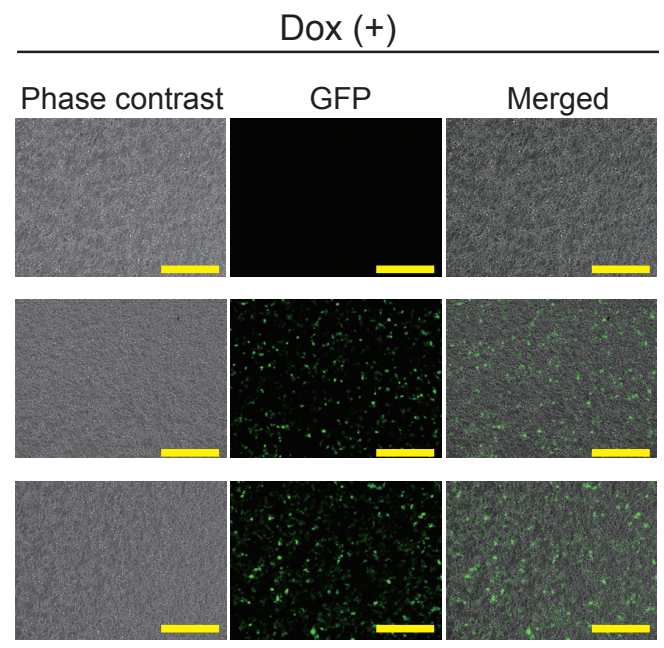

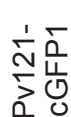
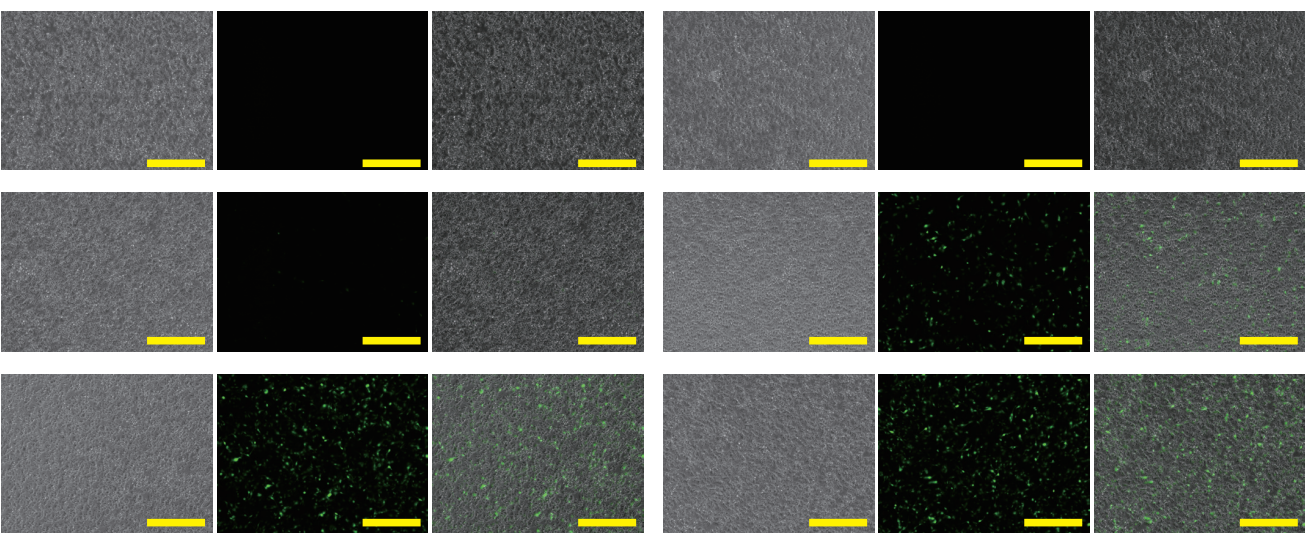

B

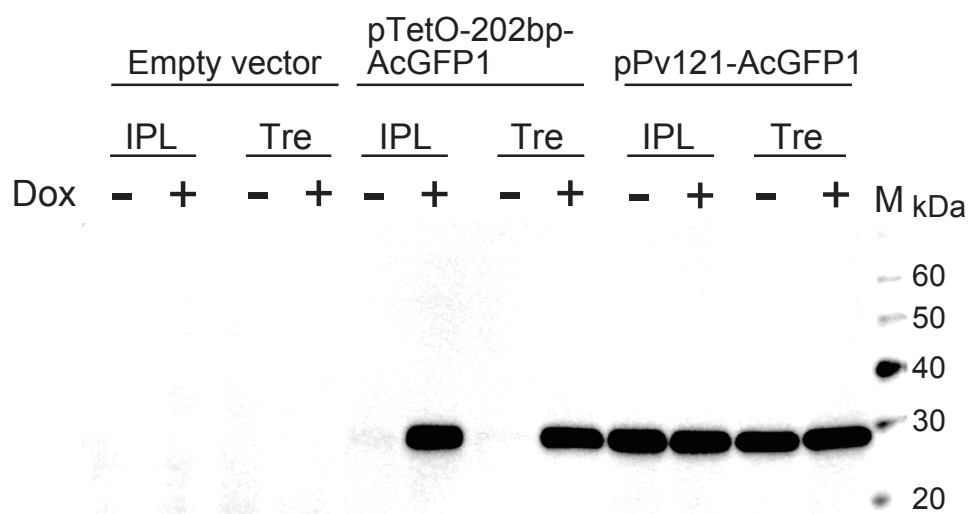

Figure 2. AcGFP1 expression using the Tet-On system in Pv11 cells. (A) The images of the cells were acquired using a BZ-X700 fluorescence microscope. The upper and lower images show the cells cultured in IPL-41 medium and $600 \mathrm{mM}$ trehalose solution, respectively. Scale bars, $200 \mu \mathrm{m}$. (B) Western blot analysis was performed on extracts of Pv11 cells transfected with the indicated vectors. IPL, IPL-41 medium; Tre, $600 \mathrm{mM}$ trehalose solution; Lane M, protein marker. 
Accordingly, we prepared the pTetO-202bp-AMV RT $\alpha$ vector as described in the Methods, and transfected this into Pv11 cells, alongside pPv121-MCS and pPv121-AMV RT $\alpha$ as negative and positive controls, respectively. As shown in Figure 3A, AMV RT $\alpha$ was observed to accumulate under Dox control for the Tet-On vector both in IPL and trehalose culture conditions, while no AMV RT $\alpha$ protein was detected with the empty vector. On the other hand, transfection with the pPv121-AMV RT $\alpha$ vector allowed for the production of AMV RT $\alpha$ protein independently of the medium or the presence of Dox (Figure 3A and Figure S2). Next, RT activity was assessed. As shown in Figure 3B, the RT activity was significantly higher in Dox-treated cells than in untreated counterparts using the Tet-On expression system, while the constitutive expression vector yielded high RT activity independently of culture conditions (Figure 3B and Table S4). These results clearly show that Pv11 cells can produce a large and complex enzyme, such as AMV RT $\alpha$, using the Tet-On inducible expression system.
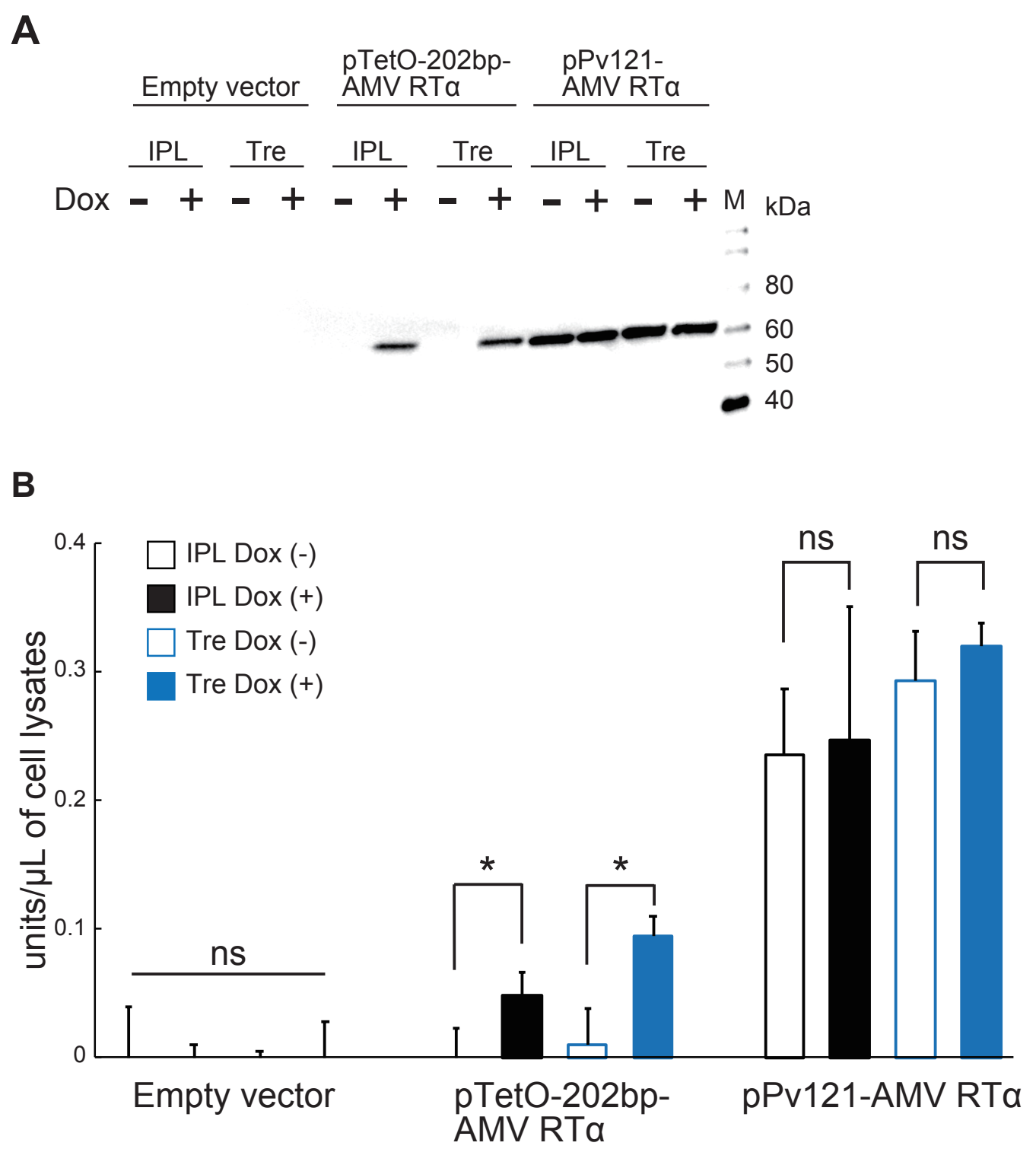

Figure 3. Avian myeloblastosis virus reverse transcriptase $\alpha$ subunit (AMV RT $\alpha$ ) expression and direct measurement of reverse transcriptase activity. (A) Western blot analysis was performed on extracts of Pv11 cells transfected with the indicated vectors. IPL, IPL-41 medium; Tre, $600 \mathrm{mM}$ trehalose solution; Lane M, protein marker. (B) AMV RT $\alpha$ activities were measured directly in lysates of Pv11 cells transfected with the indicated vectors. Normalized values are expressed as mean \pm standard deviation (SD). * , significant at $p<0.05 ; \mathrm{ns}$, not significant; $\mathrm{n}=3$ in each group. 


\section{Discussion}

Although a constitutive expression system was previously established in the anhydrobiotic cell line, Pv11 [15], no inducible system has been available so far. The present study identified a minimal promoter for Pv11 cells and applied it in the Tet-On inducible expression system. Luciferase and GFP proteins were successfully and inducibly expressed in this novel system under both regular and trehalose-treatment culture conditions. Furthermore, we showed that the AMV RT $\alpha$ protein could be inducibly expressed in Pv11 cells without losing its RT activity. This highlights the potential of Pv11 cells for the expression of larger and more complex proteins than reporter proteins and shows that Pv11 cells could have novel biotechnological applications.

The establishment of a Tet-On inducible expression system in Pv11 cells could accelerate the clarification of the molecular mechanisms underlying anhydrobiosis in this cell type and more generally. For example, the Tet-On expression system has been used for inducible gene knockdown or knockout [36-40], especially when null mutants show lethality. Such tightly regulated loss-of-function experiments will be helpful in examining whether or not a gene contributes to anhydrobiosis in Pv11 cells. Furthermore, minimal promoters constitute an essential component of massively parallel reporter assays [41-44], which are among the most powerful genome-wide screening systems. Thus, combining these cutting-edge molecular biological technologies with our current findings should result in a markedly improved understanding of the mechanisms of anhydrobiosis in Pv11 cells.

Several studies have shown that the mammalian CMV and SV40 promoters do not function in insect cell lines derived from Drosophila and Spodoptera species [30,31]. In the present study, we found that the CMV minimal promoter also does not function in Pv11 cells (Figure 1A,B). This result can probably be explained by sequence differences in the core promoter elements. The Inr in the P. vanderplanki 202 bp-promoter is $\mathrm{TCA}_{+1} \mathrm{GTT}$, whereas in the CMV minimal promoter the Inr is $\mathrm{TCA}_{+1}$ GATC (Figure 1C). Indeed, the consensus sequence for the Inr is $Y_{Y A} A_{+1} \underline{N Y}$ for humans while it is $\mathrm{YYA}_{+1} \mathrm{KTY}$ for Drosophila [23], indicating that the Inr of P. vanderplanki is very similar to that of Drosophila. This data suggests that a promoter including the Inr of humans does not work in P. vanderplanki. Clearly, the selection of a promoter with sequence elements that function in the target host cell is essential; in this context, the use of an endogenous promoter is particularly appropriate.

To develop the inducible expression system described here, we needed to define a minimal promoter and demonstrate its application. Previously, we identified 266, 202, and 137 bp fragments from the P. vanderplanki 121-promoter as candidate minimal promoters for Pv11 cells [15]. We tested the first two of these fragments, and found the $202 \mathrm{bp}$ fragment to perform well in the Tet-On system. However, it was difficult to obtain sufficient quantities of the plasmid harboring the $137 \mathrm{bp}$ fragment for expression experiments (Figure S3A,B), probably due to replication interference caused by its stem-loop structure (Figure S3C). Thus, the $137 \mathrm{bp}$ fragment was excluded from our experiments.

Several studies have shown that the maximum transcriptional activity of the Tet-On inducible expression system is comparable to that of constitutive counterparts [18]. In this study, however, the induced Nluc activity of the Tet-On system was only about 30-50\% of that of the 121-promoter under IPL $(8.88 \pm 3.05$ relative luciferase activity (RLA) vs. $26.50 \pm 16.05$ RLA) and trehalose $(8.45 \pm 0.99$ RLA vs. $16.14 \pm 7.57 \mathrm{RLA}$ ) culture conditions (Figure $1 \mathrm{~A}, \mathrm{~B}$ ). This is also reflected in the different relative AMV RT $\alpha$ activities observed between the constitutive and Tet-On inducible expression systems (Figure 3B). These results probably reflect the need to co-transfect the Tet-On 3G expression and TetO reporter vectors, both of whose expression are controlled by TetO sequences, while only one vector is sufficient to express the gene-of-interest in the constitutive expression system. In particular, in our experiments we used two vectors (e.g., pPv121-Tet-On 3G and pTetO-202bp-Nluc) for the Tet-On expression system and one vector (pPv121-Nluc) for the constitutive expression system. To reduce the complexity of the Tet-On system, and thus potentially improve expression levels, we are attempting to establish Pv11 cells that stably express the Tet-On 3G transactivator protein.

For industrial protein production, the bacterium Escherichia coli is one of the most extensively used prokaryotic cells; however, eukaryotic cells must be used instead when the protein-of-interest involves 
complex post-translational modifications and/or has multiple structural domains that are essential for its function $[45,46]$. Indeed, the expression of eukaryotic multi-domain proteins in E. coli often results in non-functional products due to differences in folding machinery and post-translational modifications between prokaryotes and eukaryotes [45,46]. Additionally, it is known that certain RT variants cannot be expressed as active or soluble heterodimers in E. coli without additional expression of appropriate molecular chaperones [47,48]. Indeed, in our experience (unpublished results), AMV RT, which is a multi-domain protein (comprising finger, palm, thumb, connection, and RNase H domains) [35], is difficult to obtain in a soluble form in sufficient quantities in E. coli. In contrast, we demonstrated in the present study that AMV RT $\alpha$ can be expressed in Pv11 cells without losing its specific activity. This result suggests that it should be possible to express a variety of proteins, including large multi-domain proteins, in Pv11 cells.

We used a simplified method here for detecting AMV RT $\alpha$ activity from crude cell lysates. Although a certain amount of nonspecific background fluorescence from the lysates of Pv11 cells cannot be avoided, this method enabled us to decrease sample volumes markedly: Transfected cells were harvested from individual wells of a 48-well plate, and only $1 \mu \mathrm{L}$ of each lysate was required for the enzyme assay. Although further improvements in our method may be necessary, it could be adapted for easy screening of useful AMV RT mutants.

In the current study, we showed the potential of Pv11 cells for various types of protein expression; however, the potential for dry preservation of proteins such as AMV RT $\alpha$ remains to be investigated. Recently, we reported the construction of a genome editing technique using CRISPR/Cas9 system [49]. By using the technique, inducible expression of AMV RT $\alpha$ in the Pv11 cell line could be established, and we will examine the possibility of preserving exogenously expressed AMV RT $\alpha$ in dried Pv11 cells.

\section{Conclusions}

The identification of a minimal promoter for Pv11 cells led to the establishment of a Tet-On inducible expression system in Pv11 cells. We showed that, in addition to the reporter proteins luciferase and AcGFP1, AMV RT $\alpha$ could be expressed successfully in the Pv11 Tet-On system without losing its RT activity. These results show that Pv11 cells have significant potential for bioindustrial protein production.

Supplementary Materials: The following are available online at http://www.mdpi.com/2075-4450/11/11/781/s1, Figure S1: The map of pPv121-MCS vector, Figure S2: Validation of western blotting analysis in Figures 2B and 3A, Figure S3: The $137 \mathrm{bp}$ fragment from the 121-promoter and its secondary structure, Table S1: Primers for the construction of AcGFP1, Nluc, and AMV RT $\alpha$ expression vectors, Table S2: The combination of transfected vectors in each experiment, Table S3: Statistical analysis of luciferase activities of cells transfected with the corresponding vectors in Figure 1A, Table S4: Statistical analysis of reverse transcriptase activities of cells transfected with the corresponding vectors in Figure 3B, Data S1: The sequence of the pPv121-MCS.

Author Contributions: S.T. and Y.M. performed the experiments, analyzed the data, contributed to discussion, and wrote the manuscript. K.U., R.D., T.O., and S.K. performed the experiments. R.D. analyzed the data and wrote the manuscript. E.S., O.G., R.C., M.I., and Y.H. contributed to discussions and participated in writing the manuscript. T.K. designed the project, wrote the manuscript, and contributed to discussions. All authors have read and agreed to the published version of the manuscript.

Funding: This work was supported by Grants-in-Aid for Scientific Research (KAKENHI) Grants (numbers JP16K15073 and JP17H01511 to T.K., JP18K14472 to Y.M., JP19J12030 to S.T., and JP18H02217 to O.G.), and was also funded by a pilot program of international collaborative research (a joint call with Russia) under "Commissioned projects for promotion of strategic international collaborative research" (JPJ008837). Bioinformatics analysis was conducted with a support of grant N20-44-07002 of Russian Science Foundation (RSF).

Acknowledgments: We are grateful to Tomoe Shiratori for routine maintenance of Pv11 cells.

Conflicts of Interest: The authors declare no conflict of interest.

\section{References}

1. Brondyk, W.H. Selecting an Appropriate Method for Expressing a Recombinant Protein. Methods Enzymol. 2009, 463, 131-147. [CrossRef] [PubMed] 
2. Van Oers, M.M.; Pijlman, G.P.; Vlak, J.M. Thirty Years of Baculovirus-Insect Cell Protein Expression: From Dark Horse to Mainstream Technology. J. Gen. Virol. 2015, 96, 6-23. [CrossRef] [PubMed]

3. Felberbaum, R.S. The Baculovirus Expression Vector System: A Commercial Manufacturing Platform for Viral Vaccines and Gene Therapy Vectors. Biotechnol. J. 2015, 10, 702-714. [CrossRef] [PubMed]

4. Backovic, M.; Krey, T. Stable Drosophila Cell Lines: An Alternative Approach to Exogenous Protein Expression. Methods Mol. Biol. 2016, 1350, 349-358. [CrossRef] [PubMed]

5. Ardisson-Araújo, D.M.P.; Morgado, F.D.S.; Schwartz, E.F.; Corzo, G.; Ribeiro, B.M. A New theraphosid Spider Toxin Causes Early Insect Cell Death by Necrosis When Expressed In Vitro during Recombinant Baculovirus Infection. PLoS ONE 2013, 8, e84404. [CrossRef]

6. Cox, M.M. Recombinant Protein Vaccines Produced in Insect Cells. Vaccine 2012, 30, 1759-1766. [CrossRef]

7. Takahashi, M.; Mitsuhashi, J.; Ohtaki, T. Establishment of a Cell Line from Embryonic Tissues of the Fleshfly, Sarcophaga peregrina (Insecta, Diptera). Dev. Growth Differ. 1980, 22, 11-19. [CrossRef]

8. Watanabe, K.; Imanishi, S.; Akiduki, G.; Cornette, R.; Okuda, T. Air-Dried Cells from the Anhydrobiotic Insect, Polypedilum vanderplanki, Can Survive Long Term Preservation at Room Temperature and Retain Proliferation Potential After Rehydration. Cryobiology 2016, 73, 93-98. [CrossRef]

9. Cornette, R.; Kikawada, T. The Induction of Anhydrobiosis in the Sleeping Chironomid: Current Status of Our Knowledge. IUBMB Life 2011, 63, 419-429. [CrossRef]

10. Crowe, J.H.; Crowe, L.M.; Oliver, A.E.; Tsvetkova, N.; Wolkers, W.; Tablin, F. The Trehalose Myth Revisited: Introduction to a Symposium on Stabilization of Cells in the Dry State. Cryobiology 2001, 43, 89-105. [CrossRef]

11. Yamada, T.G.; Suetsugu, Y.; Deviatiiarov, R.; Gusev, O.; Cornette, R.; Nesmelov, A.; Hiroi, N.; Kikawada, T.; Funahashi, A. Transcriptome Analysis of the Anhydrobiotic Cell Line Pv11 Infers the Mechanism of Desiccation Tolerance and Recovery. Sci. Rep. 2018, 8, 17941. [CrossRef] [PubMed]

12. Furuki, T.; Shimizu, T.; Chakrabortee, S.; Yamakawa, K.; Hatanaka, R.; Takahashi, T.; Kikawada, T.; Okuda, T.; Mihara, H.; Tunnacliffe, A.; et al. Effects of Group 3 LEA Protein Model Peptides on Desiccation-Induced Protein Aggregation. Biochim. Biophys. Acta 2012, 1824, 891-897. [CrossRef] [PubMed]

13. Furuki, T.; Sakurai, M. Group 3 LEA Protein Model Peptides Protect Liposomes during Desiccation. Biochim. Biophys. Acta 2014, 1838, 2757-2766. [CrossRef] [PubMed]

14. Kikuta, S.; Watanabe, S.J.; Sato, R.; Gusev, O.; Nesmelov, A.; Sogame, Y.; Cornette, R.; Kikawada, T. Towards Water-Free Biobanks: Long-Term Dry-Preservation at Room Temperature of Desiccation-Sensitive Enzyme Luciferase in Air-Dried Insect Cells. Sci. Rep. 2017, 7, 6540. [CrossRef] [PubMed]

15. Miyata, Y.; Tokumoto, S.; Sogame, Y.; Deviatiiarov, R.; Okada, J.; Cornette, R.; Gusev, O.; Shagimardanova, E.; Sakurai, M.; Kikawada, T. Identification of a Novel Strong Promoter From the Anhydrobiotic Midge, Polypedilum vanderplanki, with Conserved Function in Various Insect Cell Lines. Sci. Rep. 2019, 9, 7004. [CrossRef]

16. Dhanesuan, N.; Sharp, J.A.; Blick, T.; Price, J.T.; Thompson, E.W. Doxycycline-Inducible Expression of SPARC/Osteonectin/BM40 in MDA-MB-231 Human Breast Cancer Cells Results in Growth Inhibition. Breast Cancer Res. Treat. 2002, 75, 73-85. [CrossRef]

17. Meyer-Ficca, M.L.; Meyer, R.G.; Kaiser, H.; Brack, A.R.; Kandolf, R.; Küpper, J.-H. Comparative Analysis of Inducible Expression Systems in Transient Transfection Studies. Anal. Biochem. 2004, 334, 9-19. [CrossRef]

18. Qin, J.Y.; Zhang, L.; Clift, K.L.; Hulur, I.; Xiang, A.P.; Ren, B.-Z.; Lahn, B.T. Systematic Comparison of Constitutive Promoters and the Doxycycline-Inducible Promoter. PLoS ONE 2010, 5, e10611. [CrossRef]

19. Das, A.T.; Tenenbaum, L.; Berkhout, B. Tet-On Systems for Doxycycline-inducible Gene Expression. Curr. Gene Ther. 2016, 16, 156-167. [CrossRef]

20. Karasaki, N.; Mon, H.; Takahashi, M.; Lee, J.M.; Koga, K.; Kawaguchi, Y.; Kusakabe, T. Establishment of Tetracycline-Inducible Gene Expression Systems in the Silkworm, Bombyx Mori. Biotechnol. Lett. 2008, 31, 495-500. [CrossRef]

21. Norrman, K.; Fischer, Y.; Bonnamy, B.; Sand, F.W.; Ravassard, P.; Semb, H. Quantitative Comparison of Constitutive Promoters in Human ES Cells. PLoS ONE 2010, 5, e12413. [CrossRef] [PubMed]

22. Loew, R.; Heinz, N.; Hampf, M.; Bujard, H.; Gossen, M. Improved Tet- Responsive Promoters with Minimized Background Expression. BMC Biotechnol. 2010, 10, 81. [CrossRef] [PubMed]

23. Danino, Y.M.; Even, D.; Ideses, D.; Juven-Gershon, T. The Core Promoter: At the Heart of Gene Expression. Biochim. Biophys. Acta. 2015, 1849, 1116-1131. [CrossRef] [PubMed] 
24. Shir-Shapira, H.; Sloutskin, A.; Adato, O.; Ovadia-Shochat, A.; Ideses, D.; Zehavi, Y.; Kassavetis, G.; Kadonaga, J.T.; Unger, R.; Juven-Gershon, T. Identification of Evolutionarily Conserved Downstream Core Promoter Elements Required for the Transcriptional Regulation of Fushi Tarazu Target Genes. PLoS ONE 2019, 14, e0215695. [CrossRef]

25. Theisen, J.W.M.; Lim, C.Y.; Kadonaga, J.T. Three Key Subregions Contribute to the Function of the Downstream RNA Polymerase II Core Promoter. Mol. Cell. Biol. 2010, 30, 3471-3479. [CrossRef]

26. Even, D.Y.; Kedmi, A.; Basch-Barzilay, S.; Ideses, D.; Tikotzki, R.; Shir-Shapira, H.; Shefi, O.; Juven-Gershon, T. Engineered Promoters for Potent Transient Overexpression. PLoS ONE 2016, 11, e0148918. [CrossRef]

27. Nakahara, Y.; Imanishi, S.; Mitsumasu, K.; Kanamori, Y.; Iwata, K.-I.; Watanabe, M.; Kikawada, T.; Okuda, T. Cells from an Anhydrobiotic Chironomid Survive Almost Complete Desiccation. Cryobiology 2010, 60, 138-146. [CrossRef]

28. Sogame, Y.; Okada, J.; Kikuta, S.; Miyata, Y.; Cornette, R.; Gusev, O.; Kikawada, T. Establishment of Gene Transfer and Gene Silencing Methods in a Desiccation-Tolerant Cell Line, Pv11. Extremophiles 2016, 21, 65-72. [CrossRef]

29. Sloutskin, A.; Danino, Y.M.; Orenstein, Y.; Zehavi, Y.; Doniger, T.; Shamir, R.; Juven-Gershon, T. ElemeNT: A Computational Tool for Detecting Core Promoter Elements. Transcription 2015, 6, 41-50. [CrossRef]

30. Pfeifer, T.; Hegedus, D.; Grigliatti, T.A.; Theilmann, D.A. Baculovirus Immediate-Early Promoter-Mediated Expression of the Zeocin ${ }^{\mathrm{TM}}$ Resistance Gene for Use as a Dominant Selectable Marker in Dipteran and Lepidopteran Insect Cell Lines. Gene 1997, 188, 183-190. [CrossRef]

31. Wu, T.-Y.; Liono, L.; Chen, S.-L.; Chen, C.Y.; Chao, Y.-C. Expression of Highly Controllable Genes in Insect Cells Using a Modified Tetracycline-Regulated Gene Expression System. J. Biotechnol. 2000, 80, 75-83. [CrossRef]

32. Yamada, T.G.; Hiki, Y.; Hiroi, N.F.; Shagimardanova, E.; Gusev, O.; Cornette, R.; Kikawada, T.; Funahashi, A. Identification of a Master Transcription Factor and a Regulatory Mechanism for Desiccation Tolerance in the Anhydrobiotic Cell Line Pv11. PLoS ONE 2020, 15, e230218. [CrossRef] [PubMed]

33. Kawai, Y.; Kimura, Y.; Lezhava, A.; Kanamori, H.; Usui, K.; Hanami, T.; Soma, T.; Morlighem, J.-É.; Saga, S.; Ishizu, Y.; et al. One-Step Detection of the 2009 Pandemic Influenza A(H1N1) Virus by the RT-SmartAmp Assay and Its Clinical Validation. PLoS ONE 2012, 7, e30236. [CrossRef] [PubMed]

34. Kacian, D.; Watson, K.; Burny, A.; Spiegelman, S. Purification of the DNA Polymerase of Avian Myeloblastosis Virus. Biochim. Biophys. Acta 1971, 246, 365-383. [CrossRef]

35. Konishi, A.; Nemoto, D.; Yasukawa, K.; Inouye, K. Comparison of the Thermal Stabilities of the Alphabeta Heterodimer and the Alpha Subunit of Avian Myeloblastosis Virus Reverse Transcriptase. Biosci. Biotechnol. Biochem. 2011, 75, 1618-1620. [CrossRef] [PubMed]

36. Stegmeier, F.; Hu, G.; Rickles, R.J.; Hannon, G.J.; Elledge, S.J. A Lentiviral MicroRNA-Based System for Single-Copy Polymerase II-Regulated RNA Interference in Mammalian Cells. Proc. Natl. Acad. Sci. USA 2005, 102, 13212-13217. [CrossRef] [PubMed]

37. Dow, L.E.; Fisher, J.; O’Rourke, K.P.; Muley, A.; Kastenhuber, E.R.; Livshits, G.; Tschaharganeh, D.F.; Socci, N.D.; Lowe, S.W. Inducible In Vivo Genome Editing with CRISPR-Cas9. Nat. Biotechnol. 2015, 33, 390-394. [CrossRef] [PubMed]

38. Ono, T.; Nishijima, H.; Adachi, N.; Iiizumi, S.; Morohoshi, A.; Koyama, H.; Shibahara, K.-I. Generation of Tetracycline-Inducible Conditional Gene Knockout Cells in a Human Nalm-6 Cell Line. J. Biotechnol. 2009, 141, 1-7. [CrossRef]

39. Zhang, J.; Chen, L.; Zhang, J.; Wang, Y. Drug Inducible CRISPR/Cas Systems. Comput. Struct. Biotechnol. J. 2019, 17, 1171-1177. [CrossRef]

40. Shin, K.-J.; Wall, E.A.; Zavzavadjian, J.R.; Santat, L.A.; Liu, J.; Hwang, J.-I.; Rebres, R.; Roach, T.; Seaman, W.; Simon, M.I.; et al. A Single Lentiviral Vector Platform for MicroRNA-Based Conditional RNA Interference and Coordinated Transgene Expression. Proc. Natl. Acad. Sci. USA 2006, 103, 13759-13764. [CrossRef]

41. Muerdter, F.; Boryń, Ł.M.; Arnold, C.D. STARR-Seq-Principles and Applications. Genomics 2015, 106, 145-150. [CrossRef] [PubMed]

42. Inoue, F.; Ahituv, N. Decoding Enhancers Using Massively Parallel Reporter Assays. Genomics 2015, 106, 159-164. [CrossRef] [PubMed]

43. White, M.A. Understanding How Cis-Regulatory Function Is Encoded in DNA Sequence Using Massively Parallel Reporter Assays and Designed Sequences. Genomics 2015, 106, 165-170. [CrossRef] [PubMed] 
44. Chatterjee, S.; Ahituv, N. Gene Regulatory Elements, Major Drivers of Human Disease. Annu. Rev. Genom. Hum. Genet. 2017, 18, 45-63. [CrossRef] [PubMed]

45. Baneyx, F.; Mujacic, M. Recombinant Protein Folding and Misfolding in Escherichia coli. Nat. Biotechnol. 2004, 22, 1399-1408. [CrossRef]

46. Hartl, F.U.; Hayer-Hartl, M. Converging Concepts of Protein Folding In Vitro and In Vivo. Nat. Struct. Mol. Biol. 2009, 16, 574-581. [CrossRef]

47. Swaminathan, N. Biologically Active Reverse Transcriptases. Patent Application No. AU 26117/00A, 14 January 2000.

48. Sobek, H.; Mueller, R.; Schmidt, M.; Frey, B.; Suppmann, B.; Schmuck, R.; Thalhofer, J.-P.; Pallua, P.; Pajatsch, M. Method for Produsing an Active Heterodimeric AMV-RT in Prokaryotic Cells. U.S. Patent 6,902,920B2, 7 June 2005.

49. Miyata, Y.; Fuse, H.; Tokumoto, S.; Hiki, Y.; Deviatiiarov, R.; Yoshida, Y.; Yamada, T.G.; Cornette, R.; Gusev, O.; Shagimardanova, E.; et al. Cas9-mediated Genome Editing Reveals a Significant Contribution of Calcium Signaling Pathways to Anhydrobiosis in Pv11. bioRxiv 2020. [CrossRef]

Publisher's Note: MDPI stays neutral with regard to jurisdictional claims in published maps and institutional affiliations.

(C) 2020 by the authors. Licensee MDPI, Basel, Switzerland. This article is an open access article distributed under the terms and conditions of the Creative Commons Attribution (CC BY) license (http://creativecommons.org/licenses/by/4.0/). 